\title{
Comparative Assessment of a Self-sampling Device and Gynecologist Sampling for Cytology and HPV DNA Detection in a Rural and Low Resource Setting: Malaysian Experience
}

\author{
Latiffah A Latiff ${ }^{1,2 *}$,Zaidah Ibrahim ${ }^{1}$, Chong Pei Pei ${ }^{3}$, Sabariah Abdul Rahman $^{4,5}$, \\ Mehrnoosh Akhtari-Zavare ${ }^{1}$
}

\begin{abstract}
Purpose: This study was conducted to assess the agreement and differences between cervical self-sampling with a Kato device (KSSD) and gynecologist sampling for Pap cytology and human papillomavirus DNA (HPV DNA) detection. Materials and Methods: Women underwent self-sampling followed by gynecologist sampling during screening at two primary health clinics. Pap cytology of cervical specimens was evaluated for specimen adequacy, presence of endocervical cells or transformation zone cells and cytological interpretation for cells abnormalities. Cervical specimens were also extracted and tested for HPV DNA detection. Positive HPV smears underwent gene sequencing and HPV genotyping by referring to the online NCBI gene bank. Results were compared between samplings by Kappa agreement and McNemar test. Results: For Pap specimen adequacy, KSSD showed $100 \%$ agreement with gynecologist sampling but had only $32.3 \%$ agreement for presence of endocervical cells. Both sampling showed $100 \%$ agreement with only 1 case detected HSIL favouring CIN2 for cytology result. HPV DNA detection showed 86.2\% agreement $(\mathrm{K}=0.64,95 \% \mathrm{CI} 0.524-0.756, \mathrm{p}=0.001)$ between samplings. KSSD and gynaecologist sampling identified high risk HPV in $17.3 \%$ and $23.9 \%$ respectively (p= 0.014). Conclusion: The self-sampling using Kato device can serve as a tool in Pap cytology and HPV DNA detection in low resource settings in Malaysia. Self-sampling devices such as KSSD can be used as an alternative technique to gynaecologist sampling for cervical cancer screening among rural populations in Malaysia.
\end{abstract}

Keywords: Cervical screening - self-sampling - gynecologist sampling - HPV DNA - Pap cytology

Asian Pac J Cancer Prev, 16 (18), 8495-8501

\section{Introduction}

Cervical cancer screening (CCS) participation remain low in Malaysia and is expected to be much lower in rural area or low resource setting due to the poor knowledge about the disease (Min-Son et al., 2009; Wong, 2010). Cervical cancer (CC) ranked the third cancer among women in Malaysia with $45 \%$ women diagnosed in late stages (Ariffin et al., 2011). Study showed patients who first diagnosed with cervical cancer have a low survivability rate and even lower survivability among those women who were diagnosed at the late stages compared to early stages (Razak et al., 2013). Cervical cancer incidence among women in Malaysia increases after the age of 30 years and peaks at ages of 65-69 years with age standardized incidence rate (ASR) in this age group 30 per 100,000 (Zainal et al., 2011).

Early screening can prevent the disease, however due to economic status and unavailability of effective screening system coupled with women own personal barriers towards screening, the overall coverage of Pap smear remains low (Othman et al., 2009; Abdullah et al., 2011; Nahvijou et al., 2014).

The advent of self-sampling (SS) method for cervical screening reported to have a good acceptance among women (Alba et al., 2008; Barbee et al., 2010; Dijkstra et al., 2012) and shown to be applicable among women in low resource setting (Gok et al., 2012), or among women who never had Pap screening in their lifetime thus making the self-sampling particularly attractive for primary screening (Gravitt et al., 2008). Furthermore, there are good agreement between self-samplings and clinician samplings for the detection of HPV types and high risk HPV 13 and self-sampling shown to have high sensitivity to detect CIN2 and CIN3 when compared to standard clinician sampling (Arbyn et al., 2014).The potential use of self-sampling could benefit women in Malaysian rural area. However, there was limited information found

${ }^{1}$ Cancer Resource and Education Center (CaRE), ${ }^{2}$ Department of Community Health, ${ }^{3}$ Department of Biomedical Science, Faculty of Medicine and Health Sciences, ${ }^{4}$ Institute of Bioscience, Universiti Putra Malaysia, ${ }^{5}$ Faculty of Medicine, Universiti Teknologi Mara (UiTM), Kuala Lumpur, Malaysia*For correspondence: latiffah.latiff@gmail.com 
on self-sampling for cervical screening in Malaysia and no study ever reported on rural population and this discrepancy should be addressed. The information on self-sampling efficacy in comparison to standard sampling is highly desirable as it usages would most benefit the underserved population. To determine the self-sampling validity in rural area, here we evaluated a device by the name Kato method (Noguchi et al., 1982) in comparison with gynecologist sampling through Pap cytology and HPV DNA testing in rural area of Jempol District Negeri Sembilan Malaysia. Previously the Kato method had been severally tested for Pap cytology among women in rural of Thailand (Pengsaa et al., 1997; Pengsaa et al., 2003; Sanchaisuriya et al., 2004) and also in Japan (Noguchi et al., 1982; Okayama et al., 2012). The Kato self-sampling device (KSSD) tested in Thailand had recorded good acceptance among women in rural area (Sanchaisuriya et al., 2004), showed similar Pap cytology results (Pengsaa et al., 1997), had substantial agreement in detection of cellular changes and had moderate agreement for specimen adequacy when compared to gynecologist sampling (Pengsaa et al., 2003).

However, most studies on self-sampling in reproductive tract cytology, including KSSD, had only been used to detect cells abnormality but leaving the information on specimen's quality assessment such as the presence or absence of endocervical or transformation zone cells (EC/ $\mathrm{TZ}$ ). The presence or absence of endocervical cells or transformation zone cells (EC/TZ) in cytology report is significant to help clinician to make decision whether to repeat the Pap test or not as a part of patient management. As a matter of fact since most HPV test is feasible through self-sampling, it was also expected that KSSD to be potential for HPV tests application. Here, we hypothesized that Kato self -sampling device (KSSD) was comparable or have a good agreement with the standard clinician sampling for both Pap cytology and HPV DNA detection.

The aim of this study was to compare and seek agreement between the KSSD with gynecologist sampling as the reference standard in Pap cytology and HPV DNA test. We measured the proportions of parameters including specimen adequacy, the presence of endocervical cells or transformation zone cells (EC/TZ) and cytological interpretation of Pap cytology. For HPV DNA detection assessment, we measured and compare the proportion of HPV DNA detected, the specific HPV genotypes, high risk HPV and low risk HPV from both sampling methods.

\section{Materials and Methods}

\section{Study design}

A comparative cross sectional study was carried out from May 2010 to July 2010 among women who were referred to Primary Health Clinics, in rural District of Jempol, Negeri Sembilan, Malaysia. Participants who were pregnant, in menstruation phase and had sexual intercourse within 24 hours before screening were excluded from this study.

\section{Ethical consideration}

Ethical clearance was obtained from the Medical
Research Ethics Committee of the Faculty of Medicine and Health Sciences, University Putra Malaysia (Reference no. UPM/FPSK/PADS/T7-MJKEtikaPer/ F01(Lect(JKK)_Mac(10)05) and respondents'provided their written consent.

\section{Procedure}

A total of 486 women participated in this study after filling up consent form. Each participant agreed to provide two cervical specimens, firstly using Kato, a self-sampling devise (KSSD) after being taught and demonstrated on how to use it. In order to obtain samples, participants were asked to wash their hands, unseal the sterile device and sitting in squatted position, insert the Kato device into the vagina until it reaches the ridge on the instrument (stopper). When the device was fixed in suitable position (reached the cervix), the device was rotated 5-6 times in order to obtain the sample. Subsequently, the device was pulled out from vagina and put back in the case. The participant then handed it to the clinical staff that spreaded the sponge tip of the device onto a glass slide and fixed with $95 \%$ alcohol for cytology analysis and cut the sponge tip and put it into buffered container for HPV DNA detection.

After one hour, a second cervical specimen was performed by a gynecologist. Based on conventional technique for Pap smear cervical cytology screening in Malaysia, "Cytobrush" and the smear were spreaded onto a glass slide and fixed with $95 \%$ alcohol. The tip of cytobrush was then put into the buffered container for HPV DNA detection. These methods resulted in a total of 972 specimens.

All specimens which were obtained from Kato selfsampling device and by gynecologist were assessed, screened and interpreted by a cyto-screener technologist and a pathologist who were both blinded to the sampling techniques. The cytology was assessed according to Bethesda System 2001. The second author who was a master student performed the HPV DNA detection under the guidance of a chemical pathologist was blinded regarding the source of sampling.

\section{HPV DNA detection}

HPV DNA was performed for 226 pair specimens using the QIAmp ${ }^{\circledR}$ DNA Blood mini kit (QIAGEN®, Hilden, Germany). The purified DNA from the specimens were tested for HPV DNA through a nested (2 rounds) PCR using an outer primer (first round PCR) of MY09/ MY11 and inner primers (second round PCR ) of GP5+/ GP6+ to target the L1 gene region of the HPV viral genome. As routine positive control, purified DNAs of HPV type 18 from HeLa cell lines were used whereas for negative control, molecular grade pure water was used instead of template DNA. Reagents volume prepared for first round PCR and second round PCR per reaction were as follows: $2.5 \mathrm{ul}$ of PCR Buffer (10X), $3.0 \mathrm{ul}$ of $2.5 \mathrm{mM}$ $\mathrm{MgCl} 2,0.5 \mathrm{ul}$ of $10 \mathrm{mM} \mathrm{dNTP}, 0.5 \mathrm{ul}$ forward primers (10pmol/ul), $0.5 \mathrm{ul}$ reverse primers (10pmol/ul), $325 \mathrm{ul}$.

PCR amplification was conducted with PCR presetting of initial denaturation at $94^{\circ} \mathrm{C}$ for $45 \mathrm{sec}$, annealing at $49^{\circ} \mathrm{C} 45 \mathrm{sec}$, elongation at $72^{\circ} \mathrm{C}$ for $1 \mathrm{~min} \mathrm{sec}$ and final 
elongation at $72^{\circ} \mathrm{C}$ for $5 \mathrm{~min}$. The PCR reaction was carried out for 29 cycles of denaturation, annealing and elongation and 1 last cycle of final elongation. The results were viewed using gel electrophoresis of $1.5 \%$ agarose gel.

Following the first PCR round, all PCR products from the first round underwent the second (nested) PCR round with the same PCR setting except for annealing temperature was changed at 510C for 45 seconds. Again, the product of nested PCR underwent gel electrophoresis and the gel was photographed. A positive HPV DNA was visualized by detected band at $450 \mathrm{bp}$ in the MY09/ MY11 lane for first round PCR and/or a $150 \mathrm{bp}$ band in the GP5+/6+ in second round PCR. Re-amplification of PCR was performed on any HPV positive sample, using 3ul DNA templates from the second round PCR and double amount of the same reagents and the same presetting as in second round PCR. Afterward, gel electrophoresis using the same method was applied. Purification of the PCR product in the gel was done using QIAamp ${ }^{\circledR}$ Gel Extraction Kit (Qiagen, Germany) following the manufacturer's manual. Consecutively, the purified amplicons PCR products underwent automated DNA sequencing where both antisense and sense strands were sequenced separately from each amplicon. Using the online Basic Local Alignment Search Tool (BLAST)

Table 1. Demographic Characteristics of Women in the Study Screened for Pap Cytology and HPV DNA

\begin{tabular}{lrrrrr}
\hline Characteristics & \multicolumn{2}{c}{ Pap cytology } & \multicolumn{2}{c}{ HPV test } \\
& \multicolumn{2}{c}{$\mathrm{N}=486$} & & $\mathrm{~N}=226$ \\
\cline { 2 - 3 } \cline { 5 - 6 } & $\mathrm{n}$ & $(\%)$ & & & $(\%)$ \\
\hline Race (ethnicity) & & & & \\
$\quad$ Malay & 233 & $(47.9)$ & 226 & $(100)$ \\
Chinese & 235 & $(48.4)$ & - & - \\
Indian & 18 & $(3.7)$ & & - \\
Age group (years) & & & & \\
20-29 & 44 & $(9.1)$ & 23 & $(10.1)$ \\
$30-39$ & 92 & $(18.9)$ & 39 & $(17.2)$ \\
40-49 & 199 & $(40.9)$ & 115 & $(50.7)$ \\
$50-59$ & 123 & $(25.3)$ & 48 & $(21.1)$ \\
$>60$ & 28 & $(5.7)$ & 2 & $(0.8)$ \\
Menopausal status & & & & \\
$\quad$ Non Menopause & 390 & $(80.2)$ & 205 & $(93)$ \\
Menopause & 96 & $(19.8)$ & 21 & $(9.7)$ \\
Employment & & & & \\
Housewife & 413 & $(85)$ & 203 & $(90)$ \\
Employed & 73 & $(15)$ & 23 & $(10)$ \\
\hline
\end{tabular}

software for nucleotide, the aligned nucleotide sequences were analyzed and compared it with genomes deposited in the National Centre for Biotechnology Information (NCBI) gene bank database (http://www.ncbi.nlm.nih. gov/blast/) to identify specific genotype of HPV.

\section{Data analysis}

Analysis was performed by using SPSS Statistics 20.0 program. Descriptive statistics (Median, frequencies and percentages) were obtained for all continuous and categorical variables. The analysis was made on dichotomous data by comparing proportion for each of the parameters: I. Specimen adequacy assessment as either satisfactory or unsatisfactory for slide evaluation; II. Endocervical/transformation zone cells as either presence or absence of the cells and III. The cytology interpretation as either positive or negative for cell abnormality with positive set at result shown atypical squamous cells of undetermined significance and above (ASCUS+) or negative (normal) if result is below ASCUS (Solomon et al., 2004). Similarly, proportion of positive or negative was measured on parameters: I. HPV DNA, II. Specific HPV genotypes, III. HRHPV and IV. LRHPV. The concordant and discordant results between Kato self-sampling device and gynecologist sampling were evaluated using Cohen's Kappa. Interpretation on the kappa score ( $\mathrm{k}=0.00$ to 1.00$)$ were according to Landis and Koch (1977). Whereas, the difference in the discordant paired samples were determined by Mc-Nemar Chi-Square test. Significance level was set as $\alpha<0.05$ for all the variables in the analysis.

\section{Results}

\section{Socio-demographic characteristics}

There were 486 paired Pap cytology slides (total 972 slides) and 226 paired (total 452) of HPV DNA specimens. The median age for Pap cytology sample was 46 years old (range 21-71 years old) while for HPV test 45 years old (range 20-70 years old). The proportion of women in viable screening age (30-59 years old) for HPV test was $89 \%$ and $85 \%$ for Pap cytology test. The sociodemographic characteristics of respondents are described in detail in Table 1.

Concordance of Pap cytology

The agreement of Pap cytology result between KSSD

Table 2. Concordance of Pap Cytology of Kato Self-sampling Compared to Gynaecologist Sampling

\begin{tabular}{|c|c|c|c|c|}
\hline \multirow[b]{2}{*}{ Kato self-sampling } & \multicolumn{2}{|c|}{$\begin{array}{l}\text { Gynecologist sampling } \\
(\%)\end{array}$} & \multirow[t]{2}{*}{$\begin{array}{l}\text { Concordance } \\
(95 \% \mathrm{CI})\end{array}$} & \multirow[t]{2}{*}{$\mathrm{k}$} \\
\hline & $\mathrm{n}(\%)$ & $\mathrm{n}(\%)$ & & \\
\hline Specimen adequacy & Satisfactory & Unsatisfactory & & \\
\hline Satisfactory & $486(100)$ & $0(0)$ & $100 \%$ & - \\
\hline Unsatisfactory & $0(0)$ & $0(0)$ & & \\
\hline Endocervical cells /TZ cells & Present & Absent & & \\
\hline Present & $110(22.6)$ & $4(0.8)$ & $31.2 \%$ & 0.03 \\
\hline Absent & $330(68.0)$ & $42(8.6)$ & & $(0.02-0.05)$ \\
\hline Cytological interpretation for cell abnormality & Positive & Negative & & \\
\hline Positive & $1(0.2)$ & $0(0)$ & $100 \%$ & - \\
\hline Negative & $0(0)$ & $485(99.8)$ & & \\
\hline
\end{tabular}

*Undefined as sampling population is homogenous or low heterogeneity in categories (eg. Specimen adequacy: 100\% concordance with 100\% satisfactory category) 
Latiffah A Latiff et al

and GS was shown in Table 2. There were 100\% agreement between self-sampling and gynaecology sampling for measuring specimen adequacy and cytology interpretation cell's abnormality. One woman $(0.2 \%)$ was found positive with high grade squamous intra-epithelial lesion (HSIL) favouring CIN2. The concordance value on comparing presence of endo-cervical cells or/and transformation zone (EC/TZ) cells, were 31.2\%. Regarding endo-cervical cells /TZ cells, $68 \%$ of samples from Kato device were absent of EC/TZ but present in gynaecologist sampling. Total slides presented with EC/TZ in the KSSD and GS were $23.4 \%$ and $90.5 \%$ respectively $(\mathrm{p}=0.01)$.

\section{Concordance of HPV DNA genotype}

Table 3 shows concordance of HPV DNA, HRHPV, LRHPV and HPV genotype detection between selfsampling (KSSD) and gynecologist sampling (GS). The agreement between the two techniques in terms of detection of HPV DNA was $86.2 \%$ (Kappa=0.6; 95\% CI $0.5-0.7), 22.6 \%$ and $27 \%$, HPV positives found in self-sampling and gynecologist sampling respectively ( $\mathrm{p}=0.07$ ). We set HRHPV positive for all HRHPV types and HRHPV negative for HPV negative or/and LRHPV and found total concordance was $85.4 \%$ in sample pairs.

There were $30(13.3 \%)$ concordant pairs of HRHPV positive however were not necessarily identical in specific HPV genotypes. Among 24 discordant pairs of HRHPV positive in GS, there were $19 \mathrm{HPV}$ negative and $5 \mathrm{LRHPV}$ in KSSD. In contrast, among 9 pairs of HRHPV positive samples in KSSD, only 7 HPV negatives and 2 LRHPV in GS. Data showed HRHPV detected in KSSD and GS were $17.3 \%$ and $23.9 \%(\mathrm{p}=0.01)$ respectively. However for LRHPV detection, KSSD and GS demonstrated 5.3\% and $3.5 \%(\mathrm{p}=0.3)$. Both sampling detected 6 HPV genotypes with 5 high risk HPVs (HRHPV) type 16,18,31,33,45 and one low risk HPV (LRHPV) type 11. HPV 18 showed

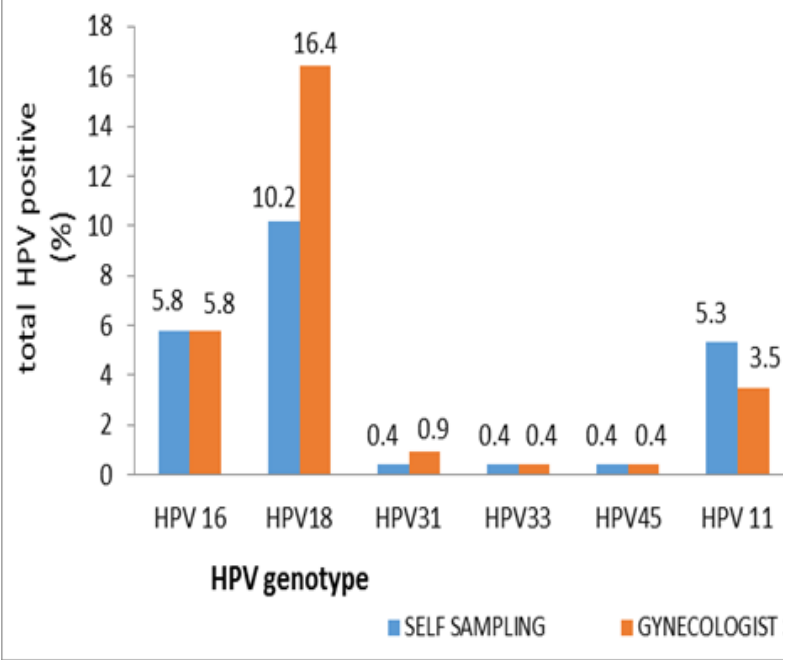

Figure 1.Comparison of Proportion of Specific HPV Type detected between Self-sampling and Gynecologist Sampling • Note: HPV $16(\mathrm{p}=1.00)$, HPV $18(\mathrm{p}=0.02)$, HPV 31 $(\mathrm{p}=1.00)$, HPV $33(\mathrm{p}=1.00)$, HPV $45(\mathrm{p}=1.00), \operatorname{HPV} 11(\mathrm{p}=0.39)$ (McNemar Chi Suare test)

Table 3. Concordance of HPV DNA Detection of Kato Self-sampling Compared to Gynecologist

\begin{tabular}{|c|c|c|c|c|}
\hline \multirow[b]{2}{*}{ Kato Self-sampling } & \multicolumn{2}{|c|}{ Gynecologist sampling } & \multirow{2}{*}{$\begin{array}{c}\text { Concordance } \\
(\%)\end{array}$} & \multirow{2}{*}{$\begin{array}{c}\mathrm{k} \\
(95 \% \mathrm{CI})\end{array}$} \\
\hline & $\begin{array}{c}\text { Positive } \\
\text { n (\%) }\end{array}$ & $\begin{array}{c}\text { Negative } \\
\mathrm{n}(\%)\end{array}$ & & \\
\hline \multicolumn{5}{|l|}{ HPV Detected } \\
\hline Positive & $41(18.1)$ & $10(4.4)$ & & 0.6 \\
\hline Negative & $21(9.3)$ & $154(68.1)$ & $86.20 \%$ & $(0.5-0.7)$ \\
\hline \multicolumn{5}{|l|}{ HR HPV } \\
\hline Positive & $30(13.3)$ & $9(3.98)$ & & 0.5 \\
\hline Negative & $24(10.6)$ & $163(72.1)$ & $85.40 \%$ & $(0.4-0.6)$ \\
\hline \multicolumn{5}{|l|}{ LR HPV } \\
\hline Positive & $4(1.8)$ & $8(3.5)$ & $93.70 \%$ & 0.3 \\
\hline Negative & $4(1.8)$ & $210(92.9)$ & & $(0.0-0.6)$ \\
\hline \multicolumn{5}{|l|}{ HPV 16} \\
\hline Positive & $5(2.2)$ & $8(3.5)$ & $93.00 \%$ & 0.3 \\
\hline Negative & $8(3.5)$ & $205(90.8)$ & & $(0.1-0.5)$ \\
\hline \multicolumn{5}{|l|}{ HPV 18} \\
\hline Positive & $14(6.2)$ & $9(4.0)$ & $86.00 \%$ & 0.3 \\
\hline Negative & $23(10.0)$ & $180(79.8)$ & & $(0.2-0.5)$ \\
\hline \multicolumn{5}{|l|}{ HPV 31} \\
\hline Positive & $0(0)$ & $1(0.5)$ & $98.60 \%$ & - \\
\hline \multicolumn{5}{|l|}{ HPV 33} \\
\hline Positive & $0(0)$ & $1(0.5)$ & $99.00 \%$ & - \\
\hline Negative & $1(0.5)$ & $224(99.0)$ & & \\
\hline \multicolumn{5}{|l|}{ HPV 45} \\
\hline Positive & $0(0)$ & $1(0.5)$ & $99.00 \%$ & - \\
\hline Negative & $1(0.5)$ & $224(99.0)$ & & \\
\hline \multicolumn{5}{|l|}{ HPV 11} \\
\hline Positive & $4(1.8)$ & $8(3.5)$ & $93.70 \%$ & 0.3 \\
\hline Negative & $4(1.8)$ & 210 (92.9) & & $(0.0-0.6)$ \\
\hline
\end{tabular}

*Table showing concordance detection of total HPV DNA, HRHPV and LRHPV and specific HPV genotype. Positive: detected, Negative: not detected. - Undefined as very low prevalence found for HPV type 31, 33 and 45 
most frequent, followed by HPV 16 in both samplings with HPV type 16/18 detected by KSSD and GS in $70.6 \%$ and $80.7 \%$. In assessing concordance of specific HPV genotype, we set HPV positive if detected and negative if not detected or other type was detected. The range of concordance between the two samplings for specific HPV genotype (HPV type $11,16,31,33,45$ ) were $86 \%$ to $99 \%$, with fair agreement were found for HPV type 16, 18 and 11 (average $\mathrm{k}=0.3$ ).

Figure 1 shows the difference in specific HPV types. Based on the result, there were no significant differences in proportion of all HPV except HPV 18.

\section{Discussion}

We studied the validity of the self-sampling device (Kato self-sampling device-KSSD) in comparison with gynecologist sampling (GS) for Pap cytology and HPV DNA testing among women in rural setting. Based on Table 2 , the cytological tests from the two different methods of collective cervical smear are generally in agreement. This was an improved result compared to other studies in Thailand (kappa=0.4, p<0.001) (Pengsaa et al., 2003); and Malaysia (Kappa $=0.2, \mathrm{p}<0.001)$ (Latiffah et al., 2015) for specimen adequacy of smears. There was discrepancy in presence of EC/TZ cells between KSSD and GS (23\% vs 90\%). However, cytology interpretation was not affected by it. Furthermore, the EC/TZ absence in screening is not a risk factor in cervical cancer development (Giorgi et al., 2010). Unsatisfactory samples are an important cause of false negative and false positive interpretation and KSSD showed no unsatisfactory samples which render its good quality for cytology. Our assessment of specimen adequacy was based on the estimated cells count per view, although, in general clinical laboratory settings, the assessment may be subjective among cytologists in which, they report the reasons accordingly (Solomon et al., 2004; Herbert et al., 2007).

The presence of EC/TZ is the key for clinicians to make decision whether the Pap test should be repeated or not (Arbyn et al., 2007). Also, previous studies suggested that HPV related cervical cancer started in the endocervical or transformation zone cells area (Boon et al., 1993; Burghardt et al., 1998). Our study showed, there was low amount of EC/TZ in KSSD compared to GS. Similarly, in previous study done by Othman and Zaki, 2014 shown that different methods of self-sampling obtain various amounts of cell mixture comprising more vaginal cells and less endocervical cells. This could be explained by the few technical reasons. We suspected that the tip of the sponge part of the Kato self-sampling device might not reach the women's endocervical area during scrapping due to the position of women squatted down while inserting the device up into vagina. While in GS, women lay down on their backs with legs wide open, letting an easy endocervical cells area sampling succeeded in collecting $90 \%$ of EC/TZ. Same posture may enhance EC/TZ collection using KSSD in future sampling. We also suspected the EC/TZ in KSSD might not be transferred well to the slide and some might left attached in the sponge of KSSD. A previous study of KSSD had tried to immerse the sponge in the liquid and subsequently transferred the liquid to the slide had found better cyto-diagnosis compared to the direct preparation method (Okayama et al., 2012). This limitation suggesting that liquid based cytology may improve the cells transfer to the glass slide. However, the absence of EC/TZ in cytology is not related to the risk of getting the disease (Kivlahan et al., 1986).

The present study also provided the first data on HPV screening in rural area, which added to a very limited study, existed in Malaysia. We found KSSD had a substantial agreement in detection of overall HPV compared to GS $(86.2 \%, \mathrm{k}=0.6)$. This was parallel to the previous study which demonstrated high concordance for detection of HPV DNA $(87 \%, \mathrm{k}=0.6)$ (Petignat et al., 2007). Recent meta-analysis study of HPV self-sampling sensitivity toward CIN in primary screening found that the self-sampling device has less variation across low risk and high risk population (Arbyn et al., 2014). As we found high agreement of KSSD and GS, the KSSD may have good potential tool to be used in high risk population in predicting pre-malignant cervical lesion.

GS detected more HRHPV than in KSSD but no difference in detecting LRHPV type. Earlier study also found oncogenic type HPV (HRHPV) were more in clinician sampling than in self-collection $(34.9 \%$ versus $23.2 \%$ ) and has no difference in sampling of non-oncogenic HPV types (Baldwin et al., 2005). In contrast, the findings of the study done by Latiffah et al. (2015) revealed a substantial agreement in detecting both high risk and low risk HPV between self-sampling and physician obtained sampling ( $\mathrm{k}=0.7$ for each). Our data showed that the discrepancy of HRHPV detected between the sampling techniques was driven by the high number of positive cases in HPV type 18 in gynecologist sampling compared to self-sampling ( $37 v s .23$ specimens, $\mathrm{p}<0.05)$. In contrast, the rest of HPV types detected around similar proportion between self-sampling and gynecologist sampling (HPV16:5.8 \% vs 5.8\%, HPV 31: $0.4 \%$ vs $0.9 \%$, HPV 33:0.4\% vs 0.4\%, HPV45: $5.3 \%$ vs $3.5 \%$ respectively). Furthermore, HPV type 16, 18 and 11 results had shown fair agreement between the two collection techniques $(\mathrm{k}=0.3)$. Different proportion of HPV type 18 detected among the two collection technique could be explained by a possibility of different area of infected HPV cells being absorbed by the sponge part of self-sampling device compared to the cervical brush by gynecologist. This were evident by the different amount of endo-cervical cells present by the two sampling techniques (lower in KSSD compared to GS) suggesting more smears from vaginal area were collected by self-sampling device. However, we would like to emphasize that the most oncogenic HRHPV type 16 shown no difference between techniques. The significant finding was, HPV 16 accounted for the most prevalent type of HPV (around $50 \%$ ) in cervical cancer cases and has the higher degree of disease severity compared to HPV18 (Franceschi, 2005).

In focus of HRHPV detection in self-sampling specimens, KSSD method carries an important benefit in rural or resource poor areas (Zhao et al., 2012). Moreover self-sampling for HPV test can reduce the burden of screening workload by clinician and Pap cytology readers 
(Garland et al., 2008). Unlike clinician sampling, through HPV self-sampling, women can be screened rapidly and rescreened again if found positive with second HPV test or Pap smear or other eligible test. However, these modalities can only be successful when the self-sampling HPV screening had been incorporated into primary screening (Cuzick, 2008). Other study also showed that women who had access to healthcare or live in urban area also had not participated in the screening (Wong et al., 2009). As selfsampling method offers a convenient way of sampling, provides time-saving method (Waller et al., 2009) and eliminates the issue of privacy and shyness (Lindell et al., 2012), the benefits could be wide speeded to general population in Malaysia.

Conclusion and recommendation In this study, we found that there was a good agreement between selfsampling and physician obtained sampling in terms of Pap cytology and HPV DNA detection for cervical screening in rural or low resource setting in Malaysia. The method is potentially giving a good impact of increasing women participation in early cervical cancer screening hence provides better quality of women's life.

\section{Acknowledgements}

The authors would like to give their gratitude to the respondents who participated in this research study. The financial source of this study was supported by Universiti Putra Malaysia with grant number 04-01-07-0313RU.

\section{References}

Abdullah F, Aziz NA, Su TT (2011). Factors related to poor practice of Pap smear screening among secondary school teachers in Malaysia. Asian Pac J Cancer Prev, 12, 1347-52.

Arbyn M, Herbert A, Schenck U, et al (2007). European guidelines for quality assurance in cervical cancer screening: recommendations for collecting samples for conventional and liquid-based cytology. Cytopathol, 18, 133-9.

Arbyn M, Verdoodt F, Snijders PJF, et al (2014). Accuracy of human papillomavirus testing on self-collected versus clinician-collected samples: a meta-analysis. Lancet Oncol, 2045, 1-12.

Baldwin S, Santos C, Mendez Brown E, et al (2005). Comparison of type-specific human papillomavirus data from self and clinician directed sampling. Gynecol Oncol, 97, 612-617.

Barbee L, Kobetz E, Menard J, et al (2010). Assessing the acceptability of self-sampling for HPV among Haitian immigrant women: CBPR in action. Cancer Causes Control, 21, 421-31.

Boon M, Suurmeijer A (1993). The Pap Smear. $2^{\text {nd }}$ edn. Leiden: Coulomb Press Leyden.

Burghardt E, Pickel H, Girardi F (1998). Colposcopy Cervical Pathology. $3^{\text {rd }}$ revise. New York.

Cuzick J, Arbyn M, Sankaranarayanan R, et al (2008). Overview of human papillomavirus-based and other novel options for cervical cancer screening in developed and developing countries. Vaccine, 26, 29-41.

DeAlba I, Anton-culver H, Hubbell FA, et al (2008). Selfsampling for human papillomavirus in a community setting: feasibility in hispanic women. Cancer Epidemiol Biomarkers Prev, 17, 2163-8.

Dijkstra MG, Heideman DAM, Van Kemenade FJ, et al (2012). Brush-based self-sampling in combination with GP5+/6+-
PCR-based hrHPV testing: high concordance with physiciantaken cervical scrapes for HPV genotyping and detection of high-grade CIN. J Clin Virol, 54, 147-151.

Franceschi S (2005). The IARC commitment to cancer prevention: the example of papillomavirus and cervical cancer. Recent Results Cancer Res, 166, 277-97.

Garland SM, Cuzick J, Domingo EJ, et al (2008). Recommendations for cervical cancer prevention in Asia Pacific. Vaccine, 26, 89-98.

Giorgi Rossi P, Baiocchi D, Ciatto S (2010). Endocervical Cells Italian Working Group. Women with a Pap test lacking endocervical cells are at lower risk of CIN2+ than women with negative Pap test with endocervical cells: a cohort study with 4.5 year follow-up. Acta Cytol, 54, 265-71.

Gok M, Heideman DAM, Van Kemenade FJ, et al (2012). Offering self-sampling for human papillomavirus testing to non-attendees of the cervical screening programme: Characteristics of the responders. Eur J Cancer, 48, 1799808.

Gravitt P, Coutlee F, Iftner T, et al (2008). New technologies in cervical cancer screening. Vaccine, 26,42-51.

Herbert A, Bergeron C, Wiener H, et al (2007). European guidelines for quality assurance in cervical cancer screening: recommendations for cervical cytology terminology. Cytopathol, 18, 213-219.

Kivlahan C, Ingra E (1986). Papanicolaou smears without endocervical cells: are they inadequate? Acta Cytol, 30, 258-60.

Latiffah AL, Sabariah AR, Wong YW, et al (2015). Assessment of the reliability of a novel self-sampling device for performing cervical sampling in Malaysia. Asian Pac J Cancer Prev, 16, 559-564.

Lindell M, Sanner K, Wikstrom I, Wilander E (2012). Self-sampling of vaginal fluid and high-risk human papillomavirus testing in women aged 50 years or older not attending Papanicolaou smear screening. BJOG, 119, 245-8.

Min-Son K, Kui SC, Spring BJ, Park S, Park EC (2009). Predicting the stage of adoption of cervical cancer screening among Korean women. Prev Med, 49, 48-53.

Nahvijou A, Hadji M, BaratiMarnani A, et al (2014). A systematic review of economic aspects of cervical cancer screening strategies worldwide: discrepancy between economic analysis and policymaking. Asian Pac J Cancer Prev, 15, 8229-37.

Noguchi M, Nakanishi M, Kato K (1982). Appraisal of a newly developed self-collection device for obtaining cervical specimens. Acta Cytol, 26, 633-635.

Okayama K, Okodo M, Fujii M, et al (2012). Improved accuracy of cytodiagnosis using the Kato self-collection devise: the usefulness of smear preparation in liquid-based cytology methods. Asian Pac J Cancer Prev, 13, 4521-4.

Othman NH, Zaki FHM (2014). Self-collection tools for routine cervical cancer screening: a review. Asian Pac J Cancer Prev, 15, 8563-9.

Othman NH, Rebolj M (2009). Challenges to Cervical Cancer Screening in a Developing Country:The Case of Malaysia. Asian Pac J Cancer Prev, 10, 747-752.

Pengsaa P, Sriamporn S, Kritpetcharat A, et al (2003). A comparison of cytology with Pap smears taken by a gynecologist and with a self-sampling device. Asian Pac J Cancer Prev, 4, 99-102.

Pengsaa P, Vatanasapt V, Sriamporn S, Sanchaisuriya P, et al (1997). A self-administered device for cervical cancer screening in northeast Thailand. Acta Cytol, 41, 749-754.

Petignat P, Faltin DL, Bruchim I, Tramèr MR, Franco EL, Coutlée F (2007). Are self-collected samples comparable to physician-collected cervical specimens for human 
papillomavirus DNA testing? A systematic review and metaanalysis. Gynecol Oncol, 105, 530-5.

Razak N, Mn K, Zubairi Y, Naing N, Zaki N (2013). Estimating the five-year survival of cervical cancer patients treated in Hospital University Sains Malaysia. Asian Pac J Cancer Prev, 14, 825-8.

Sanchaisuriya P, Pengsaa P, Sriamporn S, et al (2004). Experience with a self-administered device for cervical cancer screening by Thai women with different educational backgrounds. Asian Pac J Cancer Prev, 5,144-150.

Solomon D, Nayar R (2004). The Bethesda system for reporting cervical cytology: definitions, criteria and explanatory notes. $2^{\text {nd }}$ ed. New York: Springer.

Waller J, Bartoszek M, Marlow L, Wardle J (2009). Barriers to cervical cancer screening attendance in England: a population-based survey. J Med Screen, 16, 199-204.

Wong LP (2010). Knowledge and attitudes about HPV infection, HPV vaccination, and cervical cancer among rural south-east Asian women. Int J Behav Med, 18, 105-111.

Wong LP, Wong YL, Low WY, Khoo EM, Shuib R (2009). Knowledge and awareness of cervical cancer and screening among Malaysian women who have never had a Pap smear: a qualitative study. Singapore Med J, 50, 49-53.

Zainal Ariffin O, Nor Saleha IT (2011). National cancer registry report malaysia cancer statistics-data and figure 2007.

Zhao FH, Lewkowitz AK, Chen F, et al (2012). Pooled analysis of a self-sampling HPV DNA test as a cervical cancer primary screening method. J Natl Cancer Inst, 104, 178-88. 